\title{
ON GEODESIC MAPPINGS IN PARTICULAR CLASS OF ROTER SPACES
}

\author{
RYSZARD DESZCZ AND MARIAN HOTLOŚ \\ Dedicated to the memory of Professor Witold Roter
}

\begin{abstract}
We determine a particular class of Roter type warped product manifolds. We show that every manifold of that class admits a geodesic mapping onto a some Roter type warped product manifold. Moreover, both geodesically related manifolds are pseudosymmetric of constant type.
\end{abstract}

\section{INTRODUCTION}

Let $(M, g)$ and $(\bar{M}, \bar{g})$ be two $n$-dimensional semi-Riemannian manifolds. A diffeomorphism $h: M \rightarrow \bar{M}$ which maps geodesic lines into geodesic lines is called a geodesic transformation, or a geodesic mapping, or a projective mapping.

The well-known result of Beltrami is presented in [48, Theorem 10] as follows:

Theorem 10 (Beltrami). The real space forms constitute the projective class of the locally Euclidean spaces, or, still, by applying geodesic transformations to locally Euclidean spaces one obtains spaces of constant curvature and the class of the spaces of constant curvature is closed under geodesic transformations.

Manifolds satisfying curvature conditions and admitting geodesic transformations were investigated by several authors, see, e.g., [10, 11, 28, 29, 30, 49, 50, 56, 57, 62, 65. In particular, we have the following extension of the Beltrami's theorem [48, Theorem 19]:

Theorem 19 (Sinjukov, Mikeš, Venzi, Defever and Deszcz). If a semi-symmetric Riemannian space admits a geodesic transformation onto some other Riemann manifold, then this latter manifold must itself be pseudo-symmetric, and, if a pseudo-symmetric Riemannian space admits a geodesic transformation onto some other Riemannian manifold, then this latter manifold must itself also be pseudo-symmetric.

Thus we can state that the class of pseudosymmetric manifolds is the widest known class of manifolds which is closed with respect to geodesic mappings. It is known that the curvature tensor of certain non-conformally flat and non-quasi-Einstein pseudosymmetric manifolds of dimension $\geqslant 4$, is a linear combination of some Kulkarni-Nomizu tensors formed by the Ricci tensor and the metric tensor of the considered manifolds. A semi-Riemannian manifold with the curvature tensor having this property is named the Roter type manifold. Evidently, every

Mathematics Subject Classification (2010). Primary: 53B20. Secondary: 53C21.

Key words and phrases: geodesic mapping, warped product manifold, Einstein manifold, quasi-Einstein manifold, Roter type manifold, pseudosymmetric manifold, pseudosymmetry type curvature condition. 
Roter type manifold is pseudosymmetric. The converse statement is not true. It seems that the Roter type manifolds form an important and interesting class of manifolds for study. In particular, we can consider the following problems related to geodesic mappings of these manifolds.

(i) Does admit a Roter type manifold a geodesic mapping?

(ii) If a Roter type manifold $(M, g)$ admits a geodesic mapping onto some manifold $(\bar{M}, \bar{g})$, then in view of the above mentioned theorem $\bar{M}$ is pseudosymmetric. Therefore, it is natural to ask as follows: is $\bar{M}$ also a Roter type manifold?

In this paper we answer to these questions. First of all, we construct warped product manifolds, with 2-dimensional base and with fiber of constant curvature, which are Roter type manifolds and admit geodesic mappings. Moreover, we prove that manifolds geodesically related to these warped products are also Roter type manifolds. Furthermore, we derive some curvature conditions of pseudosymmetry type which are satisfied by constructed manifolds.

Continuing the study on geodesic mappings in Roter spaces we obtained also some new results.

\section{Preliminary Results}

Let $(M, g), n=\operatorname{dim} M \geqslant 3$, be a semi-Riemannian manifold. We denote by $\nabla, R, S, \kappa$ and $C$ the Levi-Civita connection, the Riemann-Christoffel curvature tensor, the Ricci tensor, the scalar curvature and the Weyl conformal curvature tensor of $(M, g)$, respectively. Throughout this paper all manifolds are assumed to be connected paracompact manifolds of class $C^{\infty}$.

Let $\Xi(M)$ be the Lie algebra of vector fields on $M$. We define on $M$ the endomorphisms $X \wedge_{A} Y$ and $\mathcal{R}(X, Y)$ of $\Xi(M)$ by $\left(X \wedge_{A} Y\right) Z=A(Y, Z) X-A(X, Z) Y$ and

$$
\mathcal{R}(X, Y) Z=\nabla_{X} \nabla_{Y} Z-\nabla_{Y} \nabla_{X} Z-\nabla_{[X, Y]} Z
$$

respectively, where $A$ is a symmetric $(0,2)$-tensor on $M$ and $X, Y, Z \in \Xi(M)$. The Ricci tensor $S$, the Ricci operator $\mathcal{S}$, the tensor $S^{2}$ and the scalar curvature $\kappa$ of $(M, g)$ are defined by $S(X, Y)=\operatorname{tr}\{Z \rightarrow \mathcal{R}(Z, X) Y\}, g(\mathcal{S} X, Y)=S(X, Y), S^{2}(X, Y)=S(\mathcal{S} X, Y)$ and $\kappa=\operatorname{tr} \mathcal{S}$, respectively. The endomorphism $\mathcal{C}(X, Y)$ of $(M, g), n \geq 3$, is defined by

$$
\mathcal{C}(X, Y) Z=\mathcal{R}(X, Y) Z-\frac{1}{n-2}\left(X \wedge_{g} \mathcal{S} Y+\mathcal{S} X \wedge_{g} Y-\frac{\kappa}{n-1} X \wedge_{g} Y\right) Z .
$$

The (0,4)-tensor $G$, the Riemann-Christoffel curvature tensor $R$ and the Weyl conformal curvature tensor $C$ of $(M, g)$ are defined by $G\left(X_{1}, X_{2}, X_{3}, X_{4}\right)=g\left(\left(X_{1} \wedge_{g} X_{2}\right) X_{3}, X_{4}\right)$,

$$
R\left(X_{1}, X_{2}, X_{3}, X_{4}\right)=g\left(\mathcal{R}\left(X_{1}, X_{2}\right) X_{3}, X_{4}\right), \quad C\left(X_{1}, X_{2}, X_{3}, X_{4}\right)=g\left(\mathcal{C}\left(X_{1}, X_{2}\right) X_{3}, X_{4}\right),
$$

respectively, where $X_{1}, X_{2}, X_{3}, X_{4} \in \Xi(M)$. Let $\mathcal{B}$ be a tensor field sending any $X, Y \in \Xi(M)$ to a skew-symmetric endomorphism $\mathcal{B}(X, Y)$ and let $B$ be a $(0,4)$-tensor associated with $\mathcal{B}$ by

$$
B\left(X_{1}, X_{2}, X_{3}, X_{4}\right)=g\left(\mathcal{B}\left(X_{1}, X_{2}\right) X_{3}, X_{4}\right)
$$

The tensor $B$ is said to be a generalized curvature tensor if the following conditions are satisfied

$$
\begin{aligned}
& B\left(X_{1}, X_{2}, X_{3}, X_{4}\right)=B\left(X_{3}, X_{4}, X_{1}, X_{2}\right) \\
& B\left(X_{1}, X_{2}, X_{3}, X_{4}\right)+B\left(X_{3}, X_{1}, X_{2}, X_{4}\right)+B\left(X_{2}, X_{3}, X_{1}, X_{4}\right)=0 .
\end{aligned}
$$


For $\mathcal{B}$ as above, let $B$ be again defined by (2.1). We extend the endomorphism $\mathcal{B}(X, Y)$ to a derivation $\mathcal{B}(X, Y)$. of the algebra of tensor fields on $M$, assuming that it commutes with contractions and $\mathcal{B}(X, Y) \cdot f=0$, for any smooth function $f$ on $M$. For a $(0, k)$-tensor field $T, k \geqslant 1$, we can define the $(0, k+2)$-tensor $B \cdot T$ by

$$
\begin{aligned}
& (B \cdot T)\left(X_{1}, \ldots, X_{k}, X, Y\right)=(\mathcal{B}(X, Y) \cdot T)\left(X_{1}, \ldots, X_{k}\right) \\
= & -T\left(\mathcal{B}(X, Y) X_{1}, X_{2}, \ldots, X_{k}\right)-\cdots-T\left(X_{1}, \ldots, X_{k-1}, \mathcal{B}(X, Y) X_{k}\right) .
\end{aligned}
$$

If $A$ is a symmetric $(0,2)$-tensor then we define the $(0, k+2)$-tensor $Q(A, T)$ by

$$
\begin{aligned}
& Q(A, T)\left(X_{1}, \ldots, X_{k}, X, Y\right)=\left(X \wedge_{A} Y \cdot T\right)\left(X_{1}, \ldots, X_{k}\right) \\
= & -T\left(\left(X \wedge_{A} Y\right) X_{1}, X_{2}, \ldots, X_{k}\right)-\cdots-T\left(X_{1}, \ldots, X_{k-1},\left(X \wedge_{A} Y\right) X_{k}\right) .
\end{aligned}
$$

The tensor $Q(A, T)$ is called the Tachibana tensor of the tensors $A$ and $T$, in short the Tachibana tensor (see, e.g., [17, 21, 23, 26, 27, 35]). Thus, among other things, we have the (0,6)-tensors: $R \cdot R, R \cdot C, C \cdot R, C \cdot C, Q(g, R), Q(S, R), Q(g, C)$ and $Q(S, C)$, as well as the (0,4)-tensors: $R \cdot S, C \cdot S$ and $Q(g, S)$. For a symmetric $(0,2)$-tensors $A$ and $B$ we define their Kulkarni-Nomizu product $A \wedge B$ by (see, e.g., [17, 26])

$$
\begin{aligned}
(A \wedge B)\left(X_{1}, X_{2}, X_{3}, X_{4}\right) & =A\left(X_{1}, X_{4}\right) B\left(X_{2}, X_{3}\right)+A\left(X_{2}, X_{3}\right) B\left(X_{1}, X_{4}\right) \\
& -A\left(X_{1}, X_{3}\right) B\left(X_{2}, X_{4}\right)-A\left(X_{2}, X_{4}\right) B\left(X_{1}, X_{3}\right) .
\end{aligned}
$$

A semi-Riemannian manifold $(M, g), n \geqslant 3$, is said to be an Einstein manifold (see, e.g., [2]) if at every point of $M$ its Ricci tensor $S$ is proportional to the metric tensor $g$, i.e., on $M$ we have

$$
S=\frac{\kappa}{n} g .
$$

According to [2, p. 432], (2.2) is called the Einstein metric condition. Einstein manifolds form a natural subclass of several classes of semi-Riemannian manifolds which are determined by curvature conditions imposed on their Ricci tensor [2, Table, pp. 432-433]. These conditions are named generalized Einstein curvature conditions [2, Chapter XVI].

A semi-Riemannian manifold $(M, g), n \geqslant 3$, is locally symmetric if

$$
\nabla R=0
$$

on $M$ (see, e.g., [55, Chapter 1.5]). Non-reducible locally symmetric manifolds are Einstein manifolds. The equation (2.3) implies the following integrability condition $\mathcal{R}(X, Y) \cdot R=0$, or briefly,

$$
R \cdot R=0 \text {. }
$$

Semi-Riemannian manifold satisfying (2.4) is called semisymmetric (see, e.g., [4, Chapter 8.5.3], [5. Chapter 20.7], [55, Chapter 1.6], 64, 68]). Semisymmetric manifolds form a subclass of the class of pseudosymmetric manifolds. A semi-Riemannian manifold $(M, g), n \geqslant 3$, is said to be pseudosymmetric if the tensors $R \cdot R$ and $Q(g, R)$ are linearly dependent at every point of $M$ [15] (see also [4, Chapter 8.5.3], [5, Chapter 20.7], [27, Chapter 6], [55, Chapter 12.4], [57, Chapter 7], [47, 48, 53, 66, 67, 68]). This is equivalent to

$$
R \cdot R=L_{R} Q(g, R)
$$


on $\mathcal{U}_{R}=\{x \in M \mid R-(\kappa /(n-1) n) G \neq 0$ at $x\}$, where $L_{R}$ is some function on this set. Examples of non-semisymmetric pseudosymmetric manifolds are presented among others in [13, 37, 41]. Let $\mathcal{U}_{S}$ be the set of all points of a semi-Riemannian manifold $(M, g), n \geqslant 3$, at which $S$ is not proportional to $g$, i.e., $\mathcal{U}_{S}=\{x \in M \mid S-(\kappa / n) g \neq 0$ at $x\}$. A semiRiemannian manifold $(M, g), n \geqslant 3$, is called Ricci-pseudosymmetric if the tensors $R \cdot S$ and $Q(g, S)$ are linearly dependent at every point of $M$ (see, e.g., [4, Chapter 8.5.3], [15, 18, 68]). This is equivalent on $\mathfrak{U}_{S} \subset M$ to

$$
R \cdot S=L_{S} Q(g, S)
$$

where $L_{S}$ is some function on this set. Every warped product manifold $\bar{M} \times_{F} \widetilde{N}$ with an 1dimensional manifold $(\bar{M}, \bar{g})$ and an $(n-1)$-dimensional Einstein semi-Riemannian manifold $(\widetilde{N}, \widetilde{g}), n \geqslant 3$, and a warping function $F$, is a Ricci-pseudosymmetric manifold (see, e.g., [4, Chapter 8.5.3], [8, Section 1], [23, Example 4.1], [29]). A semi-Riemannian manifold $(M, g)$ is said to be pseudosymmetric of constant type [3, 53, 54], resp., Ricci-pseudosymmetric of constant type [45], if the function $L_{R}$ is a constant on $\mathcal{U}_{R} \subset M$, resp., if the function $L_{S}$ is a constant on $\mathcal{U}_{S} \subset M$. Let $\mathcal{U}_{C}$ be the set of all points of a semi-Riemannian manifold $(M, g)$, $n \geqslant 4$, at which $C \neq 0$. We note that $\mathcal{U}_{S} \cup \mathcal{U}_{C}=\mathcal{U}_{R}$ (see, e.g., [17]). A semi-Riemannian manifold $(M, g), n \geqslant 4$, is said to have pseudosymmetric Weyl tensor if the tensors $C \cdot C$ and $Q(g, C)$ are linearly dependent at every point of $M$ (see, e.g., [5, Chapter 20.7], [17, 18, 23]). This is equivalent on $\mathfrak{U}_{C} \subset M$ to

$$
C \cdot C=L_{C} Q(g, C)
$$

where $L_{C}$ is some function on this set. Every warped product manifold $\bar{M} \times{ }_{F} \tilde{N}$, with $\operatorname{dim} \bar{M}=$ $\operatorname{dim} \tilde{N}=2$, satisfies (2.7) (see, e.g., [17, 18, 23] and references therein). Thus in particular, the Schwarzschild spacetime, the Kottler spacetime and the Reissner-Nordström spacetime satisfy (2.7). Recently, manifolds satisfying (2.7) were investigated among others in [17, 23, 35]. Warped product manifolds $\bar{M} \times_{F} \widetilde{N}$, of dimension $\geqslant 4$, satisfying on $\mathcal{U}_{C} \subset \bar{M} \times{ }_{F} \widetilde{N}$ the condition

$$
R \cdot R-Q(S, R)=L Q(g, C),
$$

where $L$ is some function on this set, were studied among others in [12, 23]. For instance, in [12] necessary and sufficient conditions for $\bar{M} \times_{F} \widetilde{N}$ to be a manifold satisfying (2.8) are given. Moreover, in that paper it was proved that any 4-dimensional warped product manifold $\bar{M} \times_{F} \tilde{N}$, with an 1-dimensional base $(\bar{M}, \bar{g})$, satisfies (2.8) [12, Theorem 4.1]. The warped product manifold $\bar{M} \times_{F} \widetilde{N}$, with 2-dimensional base $(\bar{M}, \bar{g})$ and $(n-2)$-dimensional space of constant curvature $(\tilde{N}, \widetilde{g}), n \geqslant 4$, is a manifold satisfying (2.7) and (2.8) [23, Theorem 7.1 (i)]. We refer to [8, 15, 17, 18, 21, 23, 27, 35, 38, 61, 66] for details on semi-Riemannian manifolds satisfying (2.5) and (2.6)-(2.8), as well as other conditions of this kind, named pseudosymmetry type curvature conditions or pseudosymmetry type conditions. It seems that (2.5) is the most important condition of that family of curvature conditions (see, e.g., [23]). We also can state that the Schwarzschild spacetime, the Kottler spacetime, the Reissner-Nordström spacetime, as well as the Friedmann-Lemaître-Robertson-Walker spacetimes are the "oldest" examples of pseudosymmetric warped product manifolds (see, e.g., [23, 27, 41, 61]). 
Investigations on semi-Riemannian manifolds $(M, g), n \geqslant 4$, satisfying (2.5) and (2.7) or (2.5) and (2.8) on $\mathcal{U}_{S} \cap \mathcal{U}_{C} \subset M$ lead to the following condition ([42, Theorem 3.2 (ii)], [31, Lemma 4.1], see also [23, Section 1])

$$
R=\frac{\phi}{2} S \wedge S+\mu g \wedge S+\frac{\eta}{2} g \wedge g
$$

where $\phi, \mu$ and $\eta$ are some functions on $\mathcal{U}_{S} \cap \mathcal{U}_{C}$. We note that if (2.9) is satisfied at a point of $\mathcal{U}_{S} \cap \mathcal{U}_{C}$ then at this point we have $\operatorname{rank}(S-\alpha g)>1$ for any $\alpha \in \mathbb{R}$. A semi-Riemannian manifold $(M, g), n \geqslant 4$, satisfying (2.9) on $\mathcal{U}_{S} \cap \mathcal{U}_{C} \subset M$ is called a Roter type manifold, or a Roter type space, or a Roter space [16, 24, 25].

Curvature properties of 2-recurrent semi-Riemannian manifolds $\left(\nabla^{2} R=R \otimes \psi\right)$ were investigated by Professor Witold Roter among others in [60]. In that paper it was shown that

$$
R=\frac{1}{2 \kappa} S \wedge S
$$

holds on some 2-recurrent manifolds [60, Theorem 1]. It seems that [60] is the first paper on manifolds satisfying (2.10). Evidently, (2.10) is a special case of (2.9) $(\mu=\eta=0)$, i.e.

$$
R=\frac{\phi}{2} S \wedge S
$$

We refer to [43, Example 3.1], [51, Section 4] and [58, Example 3.1] for results on manifolds satisfying (2.11).

Curvature properties of semi-Riemannian manifolds of dimension $\geqslant 4$ with parallel Weyl conformal curvature tensor $(\nabla C=0)$, non-conformally flat $(C \neq 0)$ and non-locally symmetric $(\nabla R \neq 0)$, were investigated among others in [14]. Such manifolds are also named essentially conformally symmetric manifolds, e.c.s. manifolds, in short. In [14] it was shown that the Weyl tensor $C$ of some e.c.s. manifolds is of the form $C=(\phi / 2) S \wedge S$. Since the scalar curvature $\kappa$ of every e.c.s. manifold vanishes, the last equation yields $R=(\phi / 2) S \wedge S+(1 /(n-2)) g \wedge S$. Thus we have (2.9) with $\mu=1 /(n-2)$ and $\eta=0$.

Roter type manifolds and in particular Roter type hypersurfaces (i.e. hypersurfaces satisfying (2.9) ), in semi-Riemannian spaces of constant curvature were studied in: [16, 17, 21, 24, 32, 37, 38, 39, 40, 44, 52]. Roter type manifolds satisfy several pseudosymmetry type curvature conditions, we have

Theorem 2.1. [18, 44] If $(M, g), n \geqslant 4$, is a semi-Riemannian Roter space satisfying (2.9) on $\mathcal{U}_{S} \cap \mathcal{U}_{C} \subset M$ then on this set we have

$$
\begin{gathered}
S^{2}=\alpha_{1} S+\alpha_{2} g, \quad \alpha_{1}=\kappa+\frac{(n-2) \mu-1}{\phi}, \quad \alpha_{2}=\frac{\mu \kappa+(n-1) \eta}{\phi}, \\
R \cdot R=L_{R} Q(g, R), \quad L_{R}=\frac{1}{\phi}\left((n-2)\left(\mu^{2}-\phi \eta\right)-\mu\right), \\
R \cdot C=L_{R} Q(g, C), \quad R \cdot S=L_{R} Q(g, S),
\end{gathered}
$$




$$
\begin{gathered}
R \cdot R=Q(S, R)+L Q(g, C), \quad L=L_{R}+\frac{\mu}{\phi}=\frac{n-2}{\phi}\left(\mu^{2}-\phi \eta\right), \\
C \cdot C=L_{C} Q(g, C), \quad L_{C}=L_{R}+\frac{1}{n-2}\left(\frac{\kappa}{n-1}-\alpha_{1}\right), \\
C \cdot R=L_{C} Q(g, R), \quad C \cdot S=L_{C} Q(g, S), \\
R \cdot C-C \cdot R=\left(\frac{1}{\phi}\left(\mu-\frac{1}{n-2}\right)+\frac{\kappa}{n-1}\right) Q(g, R)+\left(\frac{\mu}{\phi}\left(\mu-\frac{1}{n-2}\right)-\eta\right) Q(S, G), \\
C \cdot R-R \cdot C=Q(S, C)-\frac{\kappa}{n-1} Q(g, C) .
\end{gathered}
$$

Remark 2.1. (i) In the standard Schwarzschild coordinates $(t ; r ; \theta ; \phi)$, and the physical units $(c=G=1)$, the Reissner-Nordström-de Sitter $(\Lambda>0)$, and the Reissner-Nordström-anti-de Sitter $(\Lambda<0)$ metrics are given by the line element (see, e.g., 63])

$$
d s^{2}=-h(r) d t^{2}+h(r)^{-1} d r^{2}+r^{2}\left(d \theta^{2}+\sin ^{2} \theta d \phi^{2}\right), \quad h(r)=1-\frac{2 M}{r}+\frac{Q^{2}}{r^{2}}-\frac{\Lambda r^{2}}{3}
$$

where $M, Q$ and $\Lambda$ are non-zero constants.

(ii) [20, Section 6] The metric (2.16) satisfies (2.9) with

$$
\begin{aligned}
& \phi=\frac{3}{2}\left(Q^{2}-M r\right) r^{4} Q^{-4}, \quad \mu=\frac{1}{2}\left(Q^{4}+3 Q^{2} \Lambda r^{4}-3 \Lambda M r^{5}\right) Q^{-4} \\
& \eta=\frac{1}{12}\left(3 Q^{6}+4 Q^{4} \Lambda r^{4}-3 Q^{4} M r+9 Q^{2} \Lambda^{2} r^{8}-9 \Lambda^{2} M r^{9}\right) r^{-4} Q^{-4}
\end{aligned}
$$

If we set $\Lambda=0$ in (2.16) then we obtain the line element of the Reissner-Nordström spacetime, see, e.g., [46, Section 9.2] and references therein. It seems that the Reissner-Nordström spacetime is the "oldest" example of the Roter type warped product manifold.

(iii) Some comments on pseudosymmetric manifolds (also called Deszcz symmetric spaces), as well as Roter spaces, are given in [9, Section 1]: "From a geometric point of view, the Deszcz symmetric spaces may well be considered to be the simplest Riemannian manifolds next to the real space forms." and "From an algebraic point of view, Roter spaces may well be considered to be the simplest Riemannian manifolds next to the real space forms." For further comments we refer to 68 ].

A semi-Riemannian manifold $(M, g), n \geqslant 3$, is said to be a quasi-Einstein manifold if

$$
\operatorname{rank}(S-\alpha g)=1
$$

on $\mathcal{U}_{S} \subset M$, where $\alpha$ is some function on this set. Quasi-Einstein manifolds arose during the study of exact solutions of the Einstein field equations and the investigation on quasiumbilical hypersurfaces of conformally flat spaces (see, e.g., [18, 23] and references therein). Quasi-Einstein manifolds satisfying some pseudosymmetry type conditions were investigated among others in [1, 8, 17, 21, 35]. Quasi-Einstein hypersurfaces in semi-Riemannian spaces of constant curvature were studied among others in [19, 32, 36, 45], see also [4, Chapter 6.2], [5, Chapter 19.5], [6, Chapter 4.6], [18, 68] and references therein. We mention that there are different extensions of the class of quasi-Einstein manifolds. For instance we have the class of 
almost quasi-Einstein manifolds [7] as well as the class of 2-quasi-Einstein manifolds (see, e.g. [22, 23]).

\section{GeOdesic mappings}

Let $(M, g)$ and $(\bar{M}, \bar{g})$ be two $n$-dimensional semi-Riemannian manifolds and let a diffeomorphism $h: M \rightarrow \bar{M}$ be a geodesic mapping. It is known that in a common coordinate system $\left\{x^{1}, \ldots, x^{n}\right\}$, the Christoffel symbols, the curvature tensors and the Ricci tensors of $(M, g)$ and $(\bar{M}, \bar{g})$ are related by (see [62], [57, Chapter 8])

$$
\begin{aligned}
\bar{\Gamma}_{i j}^{h} & =\Gamma_{i j}^{h}+\delta_{i}^{h} \psi_{j}+\delta_{j}^{h} \psi_{i}, \\
\bar{R}_{i j k}^{h} & =R_{i j k}^{h}+\delta_{j}^{h} \psi_{i k}-\delta_{k}^{h} \psi_{i j}, \\
\bar{S}_{i j} & =S_{i j}-(n-1) \psi_{i j},
\end{aligned}
$$

where

$$
\psi_{i j}=\nabla_{j} \psi_{i}-\psi_{i} \psi_{j}, \quad \psi_{i}=\frac{1}{2(n+1)} \frac{\partial}{\partial x^{i}}\left(\log \left|\frac{\operatorname{det} \bar{g}}{\operatorname{det} g}\right|\right)
$$

We will denote by $h:(M, g) \stackrel{\psi}{\longrightarrow}(\bar{M}, \bar{g})$ a geodesic mapping of $(M, g)$ onto $(\bar{M}, \bar{g})$ and the manifolds $(M, g)$ and $(\bar{M}, \bar{g})$ will be called geodesically related. Further, a geodesic mapping $h:(M, g) \stackrel{\psi}{\longrightarrow}(\bar{M}, \bar{g})$ is called non-trivial on $M$ if the covector field $\psi$ with the local components $\psi_{i}$ is non-zero. It is also known that a manifold $(M, g)$ can be geodesically mapped into $(\bar{M}, \bar{g})$ if and only if there exists a covector field $\psi$ on $M$ which is a gradient with the property that

$$
\nabla_{k} \bar{g}_{i j}=2 \psi_{k} \bar{g}_{i j}+\psi_{i} \bar{g}_{j k}+\psi_{j} \bar{g}_{i k}
$$

We have the following theorem.

Theorem 3.1. [10, 28] If $(M, g)$ is a pseudosymmetric semi-Riemannian manifold admitting a non-trivial geodesic mapping $h$ onto a manifold $(\bar{M}, \bar{g})$ then $(\bar{M}, \bar{g})$ is also a pseudosymmetric manifold. Moreover,

$$
\psi_{i j}=L_{R} g_{i j}-L_{\bar{R}} \bar{g}_{i j}
$$

and $L_{R}=$ constant if and only if $L_{\bar{R}}=$ constant.

It is worth to noticing that the above statement was presented in the survey paper [56], but without proof.

In the paper [1] was considered manifolds satisfying $R \cdot R=L Q(S, R)$ or $R \cdot R=Q(S, R)$ admitting geodesic mappings.

Let $M$ be a 2-dimensional manifold with the metric

$$
d s^{2}=\mathfrak{a}(x) d x^{2}+\mathfrak{b}(x) d y^{2} .
$$

It is known ([49, 50], see also [57, p. 356]) that $M$ maps geodesically into $\bar{M}$ with the metric

$$
d \bar{s}^{2}=\frac{p \mathfrak{a}(x)}{(1+q \mathfrak{b}(x))^{2}} d x^{2}+\frac{p \mathfrak{b}(x)}{1+q \mathfrak{b}(x)} d y^{2},
$$


where $p \neq 0$ and $q$ are real parameters, $x$ and $y$ are common coordinates. Evidently we assume that $\mathfrak{a}(x) \neq 0, \mathfrak{b}(x) \neq 0$ and $1+q \mathfrak{b}(x) \neq 0$.

Taking into account that

$$
g_{11}=\mathfrak{a}(x), g_{22}=\mathfrak{b}(x), g_{12}=0, \bar{g}_{11}=\frac{p \mathfrak{a}(x)}{(1+q \mathfrak{b}(x))^{2}}, \bar{g}_{22}=\frac{p \mathfrak{b}(x)}{1+q \mathfrak{b}(x)}, \bar{g}_{12}=0
$$

it is easy to see that the only non-zero components of Christoffel symbols are the following

$$
\Gamma_{11}^{1}=\frac{\mathfrak{a}^{\prime}}{2 \mathfrak{a}}, \Gamma_{12}^{2}=\frac{\mathfrak{b}^{\prime}}{2 \mathfrak{b}}, \Gamma_{22}^{1}=-\frac{\mathfrak{b}^{\prime}}{2 \mathfrak{a}}
$$

where $\mathfrak{a}^{\prime}=\frac{d \mathfrak{a}}{d x}, \mathfrak{b}^{\prime}=\frac{d \mathfrak{b}}{d x}$. Moreover, the equality (3.4) is satisfied with $\psi$ given by

$$
\psi_{1}=-\frac{1}{2} \frac{q \mathfrak{b}^{\prime}}{1+q \mathfrak{b}}, \psi_{2}=0 .
$$

Since we are interested in non-trivial geodesic mappings, throughout this paper, we moreover assume that $q \neq 0$ and $\mathfrak{b}^{\prime}(x) \neq 0$.

The following lemma is useful.

Lemma 3.1. Let the metric $g$ on $\mathbb{R}^{2}=\{(x, y): x, y \in \mathbb{R}\}$ be of the form $g_{11}=\mathfrak{a}(x), g_{22}=$ $\mathfrak{b}(x), g_{12}=0$. For the Gauss curvature $\kappa_{G}$ of the metric $g$ we have $\kappa_{G}=K=$ const. if and only if

$$
\mathfrak{a}=\frac{\left(\mathfrak{b}^{\prime}\right)^{2}}{\mathfrak{b}(E-4 K \mathfrak{b})}, E \in \mathbb{R}
$$

Proof. We have (c.f. (4.2)

$$
R_{1221}=g_{11}\left(\partial_{1} \Gamma_{22}^{1}-\partial_{2} \Gamma_{21}^{1}+\Gamma_{22}^{r} \Gamma_{r 1}^{1}-\Gamma_{21}^{r} \Gamma_{r 2}^{1}\right)
$$

and, in virtue of (3.5) we obtain

$$
R_{1221}=\frac{1}{2}\left(-\mathfrak{b}^{\prime \prime}+\frac{\mathfrak{a}^{\prime} \mathfrak{b}^{\prime}}{2 \mathfrak{a}}+\frac{\left(\mathfrak{b}^{\prime}\right)^{2}}{2 \mathfrak{b}}\right) .
$$

On the other hand we have

$$
R_{1221}=\frac{\kappa}{2}\left(g_{11} g_{22}-g_{12}^{2}\right), \text { i.e. } R_{1221}=\frac{\kappa}{2} \mathfrak{a} \mathfrak{b}=\kappa_{G} \mathfrak{a} \mathfrak{b} .
$$

Thus we get

and by our assumption

$$
\kappa_{G}=-\frac{2 \mathfrak{a} \mathfrak{b} \mathfrak{b}^{\prime \prime}-\mathfrak{b} \mathfrak{a}^{\prime} \mathfrak{b}^{\prime}-\mathfrak{a}\left(\mathfrak{b}^{\prime}\right)^{2}}{4(\mathfrak{a} \mathfrak{b})^{2}}
$$

$$
2 \mathfrak{a} \mathfrak{b} \mathfrak{b}^{\prime \prime}-\mathfrak{b} \mathfrak{a}^{\prime} \mathfrak{b}^{\prime}-\mathfrak{a}\left(\mathfrak{b}^{\prime}\right)^{2}=-4 K \mathfrak{a}^{2} \mathfrak{b}^{2}
$$

So we obtain the following Bernoulli's equation with respect to the unknown function $\mathfrak{a}$

$$
\mathfrak{a}^{\prime}+\left(\frac{\mathfrak{b}^{\prime}}{\mathfrak{b}}-\frac{2 \mathfrak{b}^{\prime \prime}}{\mathfrak{b}^{\prime}}\right) \mathfrak{a}=\frac{4 K \mathfrak{b}}{\mathfrak{b}^{\prime}} \mathfrak{a}^{2}
$$

Thus standard calculation leads to the solution of the form (3.7). 


\section{WARPED PRODUCT MANIFOLDS}

Let $(\widehat{M}, \widehat{g})$ and $(\widetilde{N}, \widetilde{g}), \operatorname{dim} \widehat{M}=p, \operatorname{dim} \widetilde{N}=n-p, 1 \leqslant p<n$, be semi-Riemannian manifolds and $F$ a positive smooth function on $\widehat{M}$. The warped product $\widehat{M} \times_{F} \widetilde{N}$ of $(\widehat{M}, \widehat{g})$ and $(\widetilde{N}, \widetilde{g})$ is the product manifold $\widehat{M} \times \widetilde{N}$ with the metric tensor $g$ defined by

$$
g=\widehat{g} \times_{F} \widetilde{g}=\pi_{1}^{*} \widehat{g}+\left(F \circ \pi_{1}\right) \pi_{2}^{*} \widetilde{g}
$$

where $\pi_{1}: \widehat{M} \times \widetilde{N} \longrightarrow \widehat{M}$ and $\pi_{2}: \widehat{M} \times \widetilde{N} \longrightarrow \widetilde{N}$ are the natural projections on $\widehat{M}$ and $\tilde{N}$, respectively (see, e.g., 59] and references therein). Let $(\widehat{M}, \widehat{g})$ and $(\widetilde{N}, \widetilde{g})$ be covered by systems of charts $\left\{U ; x^{a}\right\}$ and $\left\{V ; y^{\alpha}\right\}$, respectively and let $\left\{U \times V ; x^{1}, \ldots, x^{p}, x^{p+1}=y^{1}, \ldots, x^{n}=y^{n-p}\right\}$ be a product chart for $\widehat{M} \times \widetilde{N}$. The local components $g_{i j}$ of the metric $g=\widehat{g} \times{ }_{F} \widetilde{g}$ with respect to this chart are the following $g_{i j}=\widehat{g}_{a b}$ if $i=a$ and $j=b, g_{i j}=F \widetilde{g}_{\alpha \beta}$ if $i=\alpha$ and $j=\beta$, and $g_{i j}=0$ otherwise, where $a, b, c, d, f \in\{1, \ldots, p\}, \alpha, \beta, \gamma, \delta \in\{p+1, \ldots, n\}$ and $h, i, j, k, l, m, r, s \in\{1,2, \ldots, n\}$. We will denote by hats (resp., by tildes) tensors formed from $\widehat{g}$ (resp., $\widetilde{g}$ ). The local components

$$
\Gamma_{i j}^{h}=\frac{1}{2} g^{h s}\left(\partial_{i} g_{j s}+\partial_{j} g_{i s}-\partial_{s} g_{i j}\right), \quad \partial_{j}=\frac{\partial}{\partial x^{j}},
$$

of the Levi-Civita connection $\nabla$ of $\widehat{M} \times_{F} \widetilde{N}$ are the following (see, e.g., [23])

$$
\begin{aligned}
& \Gamma_{b c}^{a}=\widehat{\Gamma}_{b c}^{a}, \quad \Gamma_{\beta \gamma}^{\alpha}=\widetilde{\Gamma}_{\beta \gamma}^{\alpha}, \quad \Gamma_{\alpha \beta}^{a}=-\frac{1}{2} \widehat{g}^{a b} F_{b} \widetilde{g}_{\alpha \beta} \\
& \Gamma_{a \beta}^{\alpha}=\frac{1}{2 F} F_{a} \delta_{\beta}^{\alpha}, \quad \Gamma_{\alpha b}^{a}=\Gamma_{a b}^{\alpha}=0, \quad F_{a}=\frac{\partial F}{\partial x^{a}} .
\end{aligned}
$$

The local components

$$
R_{h i j k}=g_{h s} R_{i j k}^{s}=g_{h s}\left(\partial_{k} \Gamma_{i j}^{s}-\partial_{j} \Gamma_{i k}^{s}+\Gamma_{i j}^{r} \Gamma_{r k}^{s}-\Gamma_{i k}^{r} \Gamma_{r j}^{s}\right)
$$

of the Riemann-Christoffel curvature tensor $R$ and the local components $S_{i j}$ of the Ricci tensor $S$ of the warped product $\widehat{M} \times{ }_{F} \widetilde{N}$ which may not vanish identically are the following:

$$
\begin{aligned}
R_{a b c d} & =\widehat{R}_{a b c d}, R_{\alpha a b \delta}=-\frac{1}{2} T_{a b} \widetilde{g}_{\alpha \delta}, R_{\alpha \beta \gamma \delta}=F \widetilde{R}_{\alpha \beta \gamma \beta}-\frac{\Delta_{1} F}{4} \widetilde{G}_{\alpha \beta \gamma \delta}, \\
S_{a b} & =\widehat{S}_{a b}-\frac{n-p}{2 F} T_{a b}, S_{\alpha \beta}=\widetilde{S}_{\alpha \beta}-\frac{1}{2}\left(\operatorname{tr}(T)+\frac{n-p-1}{2 F} \Delta_{1} F\right) \widetilde{g}_{\alpha \beta}, \\
T_{a b} & =\widehat{\nabla}_{a} F_{b}-\frac{1}{2 F} F_{a} F_{b}, \operatorname{tr}(T)=\widehat{g}^{a b} T_{a b}, \Delta_{1} F=\Delta_{1 \widehat{g}} F=\widehat{g}^{a b} F_{a} F_{b},
\end{aligned}
$$

where $T$ is the $(0,2)$-tensor with the local components $T_{a b}$. The scalar curvature $\kappa$ of $\widehat{M} \times_{F} \widetilde{N}$ satisfies the following equation

$$
\kappa=\widehat{\kappa}+\frac{1}{F} \widetilde{\kappa}-\frac{n-p}{F}\left(\operatorname{tr}(T)+\frac{n-p-1}{4 F} \Delta_{1} F\right) .
$$

Let $(\widehat{M}, \widehat{g})$ be a 2 -dimensional manifold with a metric $\widehat{g}$ given by

$$
\widehat{g}_{11}=\mathfrak{a}\left(x^{1}\right), \widehat{g}_{22}=\mathfrak{b}\left(x^{1}\right), \widehat{g}_{12}=0
$$


and $(\tilde{N}, \widetilde{g})$ be an $(n-2)$-dimensional, $n \geqslant 4$, semi-Riemannian space of constant curvature, when $n \geqslant 5$. Next let $\widehat{M} \times_{F} \widetilde{N}$ be the warped product with warping function $F=F\left(x^{1}, x^{2}\right)$. Let $(\overline{\widehat{M}}, \overline{\widehat{g}})$ be a manifold geodesically related to $(\widehat{M}, \widehat{g})$ with a metric $\overline{\widehat{g}}$ given by

$$
\overline{\widehat{g}}_{11}=\frac{p \mathfrak{a}\left(x^{1}\right)}{\left(1+q \mathfrak{b}\left(x^{1}\right)\right)^{2}}, \overline{\widehat{g}}_{22}=\frac{p \mathfrak{b}\left(x^{1}\right)}{1+q \mathfrak{b}\left(x^{1}\right)}, \overline{\widehat{g}}_{12}=0
$$

and a covector field $\psi$ such as in (3.6).

We will find the necessary and sufficient conditions that the warped product manifold $\widehat{M} \times{ }_{F} \widetilde{N}$ can be geodesically mapped into the warped product manifold $\widehat{\widehat{M}} \times \bar{F} \widetilde{N}$ with a warping function $\bar{F}=\bar{F}\left(x^{1}, x^{2}\right)$. Under our assumptions we have

$$
\begin{aligned}
g_{11} & =\mathfrak{a}\left(x^{1}\right), g_{22}=\mathfrak{b}\left(x^{1}\right), g_{\alpha \beta}=F \widetilde{g}_{\alpha \beta}, \\
\bar{g}_{11} & =\frac{p \mathfrak{a}\left(x^{1}\right)}{\left(1+q \mathfrak{b}\left(x^{1}\right)\right)^{2}}, \bar{g}_{22}=\frac{p \mathfrak{b}\left(x^{1}\right)}{1+q \mathfrak{b}\left(x^{1}\right)}, \bar{g}_{\alpha \beta}=\bar{F} \widetilde{g}_{\alpha \beta},
\end{aligned}
$$

and remaining components of $g$ and $\bar{g}$ vanish.

It is obvious that the equality (3.4) is satisfied for $i=a, j=b, k=c$.

Considering the case $i=a, j=\alpha, k=\beta$ we have, in virtue of (4.1)

$$
\begin{gathered}
\frac{\partial \bar{g}_{a \alpha}}{\partial x^{\beta}}-\Gamma_{\beta a}^{s} \bar{g}_{s \alpha}-\Gamma_{\beta \alpha}^{s} \bar{g}_{s a}=2 \psi_{\beta} \bar{g}_{a \alpha}+\psi_{a} \bar{g}_{\alpha \beta}+\psi_{\alpha} \bar{g}_{a \beta}, \\
-\Gamma_{\beta a}^{\epsilon} \bar{g}_{\epsilon \alpha}-\Gamma_{\beta \alpha}^{\epsilon} \bar{g}_{\epsilon a}-\Gamma_{\beta \alpha}^{c} \bar{g}_{c a}=\psi_{a} \bar{g}_{\alpha \beta}, \\
-\frac{1}{2 F} F_{a} \bar{g}_{\alpha \beta}+\frac{1}{2} \frac{F^{c}}{F} \bar{g}_{c a} F \widetilde{g}_{\alpha \beta}=\psi_{a} \bar{g}_{\alpha \beta}
\end{gathered}
$$

and finally

$$
-\frac{\bar{F}}{2 F} F_{a}+\frac{1}{2} F^{c} \bar{g}_{c a}=\bar{F} \psi_{a} .
$$

Now, let $i=\alpha, j=\beta, k=a$. We have in sequence

$$
\begin{gathered}
\frac{\partial \bar{g}_{\alpha \beta}}{\partial x^{a}}-\Gamma_{a \alpha}^{s} \bar{g}_{s \beta}-\Gamma_{a \beta}^{s} \bar{g}_{s \alpha}=2 \psi_{a} \bar{g}_{\alpha \beta}+\psi_{\alpha} \bar{g}_{a \beta}+\psi_{\beta} \bar{g}_{a \alpha}, \\
\frac{\partial \bar{F}}{\partial x^{a}} \widetilde{g}_{\alpha \beta}-\Gamma_{a \alpha}^{\epsilon} \bar{g}_{\epsilon \beta}-\Gamma_{a \beta}^{\epsilon} \bar{g}_{\epsilon \alpha}=2 \psi_{a} \bar{g}_{\alpha \beta}, \\
\frac{\partial \bar{F}}{\partial x^{a}}-\frac{1}{2 F} F_{a} \bar{F}-\frac{1}{2 F} F_{a} \bar{F}=2 \psi_{a} \bar{F} \\
\frac{1}{\bar{F}} \frac{\partial \bar{F}}{\partial x^{a}}=\frac{F_{a}}{F}+2 \psi_{a}, \quad \frac{\partial \log \bar{F}}{\partial x^{a}}-\frac{\partial \log F}{\partial x^{a}}=2 \psi_{a},
\end{gathered}
$$

and finally

$$
\frac{\partial}{\partial x^{a}}\left(\log \frac{\bar{F}}{F}\right)=2 \psi_{a} .
$$

It is easy to check that in the remaining cases (3.4) is also satisfied. Thus we have proved 
Proposition 4.1. Let $(\widehat{M}, \widehat{g})$ be a 2-dimensional manifold with a metric $\widehat{g}$ given by

$$
\widehat{g}_{11}=\mathfrak{a}\left(x^{1}\right), \widehat{g}_{22}=\mathfrak{b}\left(x^{1}\right), \widehat{g}_{12}=0
$$

and $(\tilde{N}, \widetilde{g})$ be an $(n-2)$-dimensional, $n \geqslant 4$, semi-Riemannian space of constant curvature, when $n \geqslant 5$. Next let $\widehat{M} \times_{F} \widetilde{N}$ be the warped product manifold with warping function $F=$ $F\left(x^{1}, x^{2}\right)$ and let $(\overline{\widehat{M}}, \overline{\widehat{g}})$ be a manifold geodesically related to $(\widehat{M}, \widehat{g})$ with a metric $\overline{\widehat{g}}$ given by

$$
\overline{\widehat{g}}_{11}=\frac{p \mathfrak{a}\left(x^{1}\right)}{\left(1+q \mathfrak{b}\left(x^{1}\right)\right)^{2}}, \overline{\widehat{g}}_{22}=\frac{p \mathfrak{b}\left(x^{1}\right)}{1+q \mathfrak{b}\left(x^{1}\right)}, \overline{\widehat{g}}_{12}=0
$$

and a covector field $\psi$ such as in (3.6). Then the warped product manifold $\widehat{M} \times_{F} \widetilde{N}$ can be geodesically mapped into the warped product manifold $\widehat{\widehat{M}} \times \bar{F} \widetilde{N}$ with a warping function $\bar{F}=\bar{F}\left(x^{1}, x^{2}\right)$ if and only if the equalities (4.6) and (4.7) are satisfied.

According to [23, Theorem 5.3] the warped product manifold $\widehat{M} \times_{F} \widetilde{N}$ with 2-dimensional manifold $(\widehat{M}, \widehat{g})$ and $(n-2)$-dimensional semi-Riemannian space of constant curvature is pseudosymmetric on the set $U_{S} \cap U_{C}$ if and only if $T_{a b}$ is proportional to $\widehat{g}_{a b}$ on this set. Therefore let the warped product manifold $\widehat{M} \times_{F} \widetilde{N}$ be such as in Proposition 4.1 with

$$
F=F\left(x^{1}, x^{2}\right)=f^{2}\left(x^{1}, x^{2}\right)
$$

and we consider now the condition: $T=\lambda \widehat{g}$. In view of (4.5) this condition is equivalent to

$$
\text { (i) } T_{12}=0, \quad(\text { ii }) \mathfrak{b} T_{11}=\mathfrak{a} T_{22} \text {. }
$$

Further, by (4.4)

$$
T_{12}=\widehat{\nabla}_{1} F_{2}-\frac{1}{2 F} F_{1} F_{2}=\partial_{1} F_{2}-F_{s} \Gamma_{12}^{s}-\frac{1}{2 F} F_{1} F_{2}=\partial_{1} F_{2}-F_{2} \Gamma_{12}^{2}-\frac{1}{2 F} F_{1} F_{2},
$$

so using (4.9)(i), (3.5) and (4.8) we get

$$
\begin{gathered}
2\left(f_{1} f_{2}+f f_{12}\right)-2 f f_{2} \frac{\mathfrak{b}^{\prime}}{2 \mathfrak{b}}-\frac{1}{2 f^{2}} 2 f f_{1} 2 f f_{2}=0, \\
f_{12}=f_{2} \frac{\mathfrak{b}^{\prime}}{2 \mathfrak{b}} .
\end{gathered}
$$

Similarly,

$$
\begin{gathered}
T_{11}=\partial_{1} F_{1}-\frac{\mathfrak{a}^{\prime}}{2 \mathfrak{a}} F_{1}-\frac{1}{2 F} F_{1}^{2}=2\left(f_{1}^{2}+f f_{11}\right)-\frac{\mathfrak{a}^{\prime}}{2 \mathfrak{a}} 2 f f_{1}-\frac{1}{2 f^{2}} 4 f^{2} f_{1}^{2}=2 f f_{11}-2 f f_{1} \frac{\mathfrak{a}^{\prime}}{2 \mathfrak{a}} \\
T_{22}=\partial_{2} F_{2}+\frac{\mathfrak{b}^{\prime}}{2 \mathfrak{a}} F_{1}-\frac{1}{2 F} F_{2}^{2}=2\left(f_{2}^{2}+f f_{22}\right)+\frac{\mathfrak{b}^{\prime}}{2 \mathfrak{a}} 2 f f_{1}-2 f_{2}^{2}=2 f f_{22}+2 f f_{1} \frac{\mathfrak{b}^{\prime}}{2 \mathfrak{a}}
\end{gathered}
$$

Thus (4.9) (ii) leads to

$$
\begin{gathered}
\mathfrak{b} f_{11}-\mathfrak{a} f_{22}=f_{1}\left(\frac{\mathfrak{b} \mathfrak{a}^{\prime}}{2 \mathfrak{a}}+\frac{\mathfrak{b}^{\prime} \mathfrak{a}}{2 \mathfrak{a}}\right)=\frac{f_{1}}{2 \mathfrak{a}}(\mathfrak{a} \mathfrak{b})^{\prime}, \\
f_{11}-\frac{\mathfrak{a}}{\mathfrak{b}} f_{22}=\frac{f_{1}}{2 \mathfrak{a} \mathfrak{b}}(\mathfrak{a} \mathfrak{b})^{\prime}
\end{gathered}
$$


Now we will find conditions for which equations (4.6) and (4.7) will be satisfied.

Using (4.6) for $a=2$, in virtue of (3.6), we have

$$
\begin{gathered}
\frac{\bar{F}}{F} F_{2}=F^{c} \bar{g}_{c 2}=F_{s} \widehat{g}^{2 s} \bar{g}_{22}=F_{2} \frac{1}{\mathfrak{b}} \frac{p \mathfrak{b}}{1+q \mathfrak{b}}=\frac{p F_{2}}{1+q \mathfrak{b}}, \\
\frac{\bar{F}}{F}=\frac{p}{1+q \mathfrak{b}} .
\end{gathered}
$$

This implies that $\frac{\partial}{\partial x^{2}}\left(\log \frac{\bar{F}}{F}\right)=0$. On the other hand $\frac{\partial}{\partial x^{1}}\left(\log \frac{\bar{F}}{F}\right)=-\frac{q \mathfrak{b}^{\prime}}{1+q \mathfrak{b}}=2 \psi_{1}$. Thus the equality (4.7) is satisfied.

The condition (4.6) for $a=1$ takes the form $-\frac{\bar{F}}{F} F_{1}+F^{c} \bar{g}_{c 1}=2 \bar{F} \psi_{1}$. Since

$$
F^{c} \bar{g}_{c 1}=F_{s} \widehat{g}^{s 1} \bar{g}_{11}=F_{1} \frac{1}{\mathfrak{a}} \frac{p \mathfrak{a}}{(1+q \mathfrak{b})^{2}}=F_{1} \frac{p}{(1+q \mathfrak{b})^{2}},
$$

so using (4.12) we get $\mathfrak{b}^{\prime} F=\mathfrak{b} F_{1}$ which in term of $f$ takes the form $2 \mathfrak{b} f_{1}=\mathfrak{b}^{\prime} f$. Applying this equality to (4.10) we have $f f_{12}=f_{1} f_{2}$. It is easy to see that the solution of this differential equation is the following $f\left(x^{1}, x^{2}\right)=A\left(x^{1}\right) B\left(x^{2}\right)$. Thus $f_{1} / f=A_{1} / A$. But $f_{1} / f=\mathfrak{b}^{\prime} /(2 \mathfrak{b})$ and we obtain $2 A_{1} / A=\mathfrak{b}^{\prime} / \mathfrak{b}$, which in particular gives $A^{2}=\mathfrak{b}$ and without loss of generality $f\left(x^{1}, x^{2}\right)=\sqrt{\mathfrak{b}\left(x^{1}\right)} B\left(x^{2}\right)$. This leads to:

$$
f_{1}=\frac{\mathfrak{b}^{\prime}}{2 \sqrt{\mathfrak{b}}} B, f_{2}=\sqrt{\mathfrak{b}} B_{2}, f_{22}=\sqrt{\mathfrak{b}} B_{22}, f_{11}=\frac{B}{2 \sqrt{\mathfrak{b}}}\left(\mathfrak{b}^{\prime \prime}-\frac{\left(\mathfrak{b}^{\prime}\right)^{2}}{2 \mathfrak{b}}\right) .
$$

Substituting these equalities into (4.11) we have

The last equality implies

$$
\begin{gathered}
\frac{B}{2 \sqrt{\mathfrak{b}}}\left(\mathfrak{b}^{\prime \prime}-\frac{\left(\mathfrak{b}^{\prime}\right)^{2}}{2 \mathfrak{b}}\right)-\frac{\mathfrak{a}}{\mathfrak{b}} \sqrt{\mathfrak{b}} B_{22}=\frac{(\mathfrak{a} \mathfrak{b})^{\prime}}{2 \mathfrak{a} \mathfrak{b}} \frac{\mathfrak{b}^{\prime} B}{2 \sqrt{\mathfrak{b}}} \\
\mathfrak{b}^{\prime \prime}-\frac{\left(\mathfrak{b}^{\prime}\right)^{2}}{2 \mathfrak{b}}=2 \mathfrak{a} \frac{B_{22}}{B}+\frac{(\mathfrak{a} \mathfrak{b})^{\prime}}{2 \mathfrak{a} \mathfrak{b}} \mathfrak{b}^{\prime}
\end{gathered}
$$

$$
\frac{B_{22}}{B}=C=\text { const }
$$

Therefore we have

$$
\mathfrak{b} \mathfrak{b}^{\prime \prime}-\left(\mathfrak{b}^{\prime}\right)^{2}=2 \mathfrak{a} \mathfrak{b} C+\frac{\mathfrak{a}^{\prime} \mathfrak{b}^{\prime} \mathfrak{b}}{2 \mathfrak{a}}
$$

Rewriting this equation in the form

$$
-\mathfrak{a}^{\prime}+2 \mathfrak{a}\left(\frac{\mathfrak{b}^{\prime \prime}}{\mathfrak{b}^{\prime}}-\frac{\mathfrak{b}^{\prime}}{\mathfrak{b}}\right)=\frac{4 C \mathfrak{a}^{2}}{\mathfrak{b}^{\prime}}
$$

we have Bernoulli's equation with respect to the unknown function $\mathfrak{a}$ which leads to

$$
\mathfrak{a}=\frac{\left(\mathfrak{b}^{\prime}\right)^{2}}{\mathfrak{b}(D \mathfrak{b}-4 C)}, D \in \mathbb{R} .
$$

Comparing this equality with Lemma 3.1 we have the following. 
Corollary 4.1. Let $(M, g)$ be a 2-dimensional manifold with the metric

$$
g_{11}=\mathfrak{a}\left(x^{1}\right), g_{22}=\mathfrak{b}\left(x^{1}\right), g_{12}=0
$$

and let functions $\mathfrak{a}$ and $\mathfrak{b}$ satisfy (4.14). Then the function $\mathfrak{a}$ satisfies the relation (4.15) and the Gauss curvature of $M$ is constant, namely

$$
\kappa_{G}=-\frac{D}{4}
$$

Taking into account the equality $f\left(x^{1}, x^{2}\right)=\sqrt{\mathfrak{b}\left(x^{1}\right)} B\left(x^{2}\right)$ and (4.8) we have

$$
F\left(x^{1}, x^{2}\right)=\mathfrak{b}\left(x^{1}\right) B^{2}\left(x^{2}\right) \text {. }
$$

Computing once more $T_{22}$ and using (4.17), (4.13) and (4.15) we obtain $T_{22}=(D / 2) \mathfrak{b}^{2} B^{2}$. Thus, in view of (4.9) (ii) we have $\operatorname{tr}(T)=\widehat{g}^{11} T_{11}+\widehat{g}^{22} T_{22}=(2 / \mathfrak{b}) T_{22}=D \mathfrak{b} B^{2}$, i.e.

$$
\operatorname{tr}(T)=D F \text {. }
$$

For a function $B$ satisfying (4.13) we have the following.

Remark 4.1. Let a function $B: \mathbb{R} \rightarrow \mathbb{R}$ satisfies $B^{\prime \prime}(t) / B(t)=C=$ const. Then:

(i) $\left(B^{\prime}\right)^{2}-C B^{2}=$ const.,

(ii) the function $B$, according to $C$, is of the form:

(a) $C>0$, then $B(t)=C_{1} e^{\sqrt{C} t}+C_{2} e^{-\sqrt{C} t}, C_{1}, C_{2} \in \mathbb{R}$,

(b) $C<0$, then $B(t)=C_{1} \cos \sqrt{-C} t+C_{2} \sin \sqrt{-C} t, C_{1}, C_{2} \in \mathbb{R}$,

(c) $C=0$, then $B(t)=C_{1} t+C_{2}, C_{1}, C_{2} \in \mathbb{R}$.

Concerning the conformal flatness of warped product manifolds we have the following.

Remark 4.2. Let $\widehat{M} \times_{F} \widetilde{N}$ be the warped product manifold with a 2-dimensional manifold $(\widehat{M}, \widehat{g})$ and an $(n-2)$-dimensional fiber $(\widetilde{N}, \widetilde{g}), n \geqslant 4$, and a warping function $F$, and let $(\tilde{N}, \widetilde{g})$ be a semi-Riemannian space of constant curvature, when $n \geqslant 5$. The local components $C_{\text {hijk }}$ of the Weyl conformal curvature tensor $C$ of $\widehat{M} \times_{F} \widetilde{N}$ are expressed by (see [23])

$$
\begin{gathered}
C_{a b c d}=\frac{(n-3) \rho_{0}}{n-1} G_{a b c d}, \quad C_{\alpha b c \delta}=-\frac{(n-3) \rho_{0}}{(n-2)(n-1)} G_{\alpha b c \delta}, \\
C_{\alpha \beta \gamma \delta}=\frac{2 \rho_{0}}{(n-2)(n-1)} G_{\alpha \beta \gamma \delta}, \quad C_{a b c \delta}=C_{a b \gamma \delta}=C_{a \beta \gamma \delta}=0, \\
\rho_{0}=\frac{\widehat{\kappa}}{2}+\frac{\widetilde{\kappa}}{(n-3)(n-2) F}+\frac{\operatorname{tr}(T)}{2 F}-\frac{\Delta_{1} F}{4 F^{2}} .
\end{gathered}
$$

Hence, $\widehat{M} \times_{F} \tilde{N}$ is conformally flat if and only if $\rho_{0}=0$.

Taking into account (4.15) we find

$$
\Delta_{1} F=\frac{1}{\mathfrak{a}} F_{1}^{2}+\frac{1}{\mathfrak{b}} F_{2}^{2}=\mathfrak{b} B^{2}\left(B^{2}(D \mathfrak{b}-4 C)+4 B_{2}^{2}\right) .
$$


Thus, by using (4.19), (4.16) and (4.18), we obtain

$$
\begin{aligned}
\rho_{0} & =-\frac{D}{4}+\frac{\widetilde{\kappa}}{(n-3)(n-2)} \frac{1}{F}+\frac{D F}{2 F}-\frac{1}{F} \frac{1}{4}\left(B^{2}(D \mathfrak{b}-4 C)+4 B_{2}^{2}\right)= \\
& =\frac{D}{4}+\frac{\widetilde{\kappa}}{(n-3)(n-2) F}-\frac{1}{F}\left(B^{2}\left(\frac{D}{4} \mathfrak{b}-C\right)+B_{2}^{2}\right)= \\
& =\frac{1}{F}\left(\frac{\widetilde{\kappa}}{(n-3)(n-2)}-B_{2}^{2}+C B^{2}\right) .
\end{aligned}
$$

Therefore, the equality $\rho_{0}=0$ is equivalent to

$$
\frac{\widetilde{\kappa}}{(n-3)(n-2)}=B_{2}^{2}-C B^{2} \text {. }
$$

Now we consider the following problem: when the manifold $\widehat{M} \times_{F} \widetilde{N}$ is quasi-Einsteinian or Einsteinian. In virtue of (4.3) we have

$$
S_{a b}=\widehat{S}_{a b}-\frac{n-2}{F} T_{a b}=\left(\frac{\widehat{\kappa}}{2}-\frac{n-2}{2 F} \frac{\operatorname{tr}(T)}{2}\right) g_{a b}
$$

and in view of $\widehat{\kappa}_{G}=\widehat{\kappa} / 2$, (4.16) and (4.18) we get

$$
S_{a b}=-\frac{n-1}{4} D g_{a b}
$$

Similarly,

$$
S_{\alpha \beta}=\widetilde{S}_{\alpha \beta}-\frac{1}{2}\left(\operatorname{tr}(T)+\frac{n-3}{2} \frac{\Delta_{1} F}{F}\right) \widetilde{g}_{\alpha \beta}=\left(\frac{\widetilde{\kappa}}{n-2}-\frac{\operatorname{tr}(T)}{2}-\frac{n-3}{4 F} \Delta_{1} F\right) \frac{1}{F} g_{\alpha \beta} .
$$

Taking into account (4.20) we find

$$
S_{\alpha \beta}=\frac{1}{\mathfrak{b} B^{2}}\left(\frac{\widetilde{\kappa}}{n-2}-\frac{n-1}{4} D \mathfrak{b} B^{2}+(n-3)\left(B^{2} C-B_{2}^{2}\right)\right) g_{\alpha \beta} .
$$

Equalities (2.17), (4.22) and (4.23) imply that $\widehat{M} \times_{F} \widetilde{N}$ cannot be quasi-Ensteinian and will be Einsteinian if and only if

$$
\frac{\widetilde{\kappa}}{n-2}-\frac{n-1}{4} D \mathfrak{b} B^{2}+(n-3)\left(B^{2} C-B_{2}^{2}\right)=-\frac{n-1}{4} D \mathfrak{b} B^{2},
$$

which reduces to the equality (4.21).

Remark 4.3. Let $\widehat{M} \times_{F} \widetilde{N}$ be the warped product manifold with a 2-dimensional manifold $(\widehat{M}, \widehat{g})$ and an $(n-2)$-dimensional fiber $(\widetilde{N}, \widetilde{g}), n \geqslant 4$, and a warping function $F$, and let $(\widetilde{N}, \widetilde{g})$ be a semi-Riemannian space of constant curvature, when $n \geqslant 5$. If $T=\frac{\operatorname{tr}(T)}{2} \widehat{g}$ on $U=U_{S} \cap U_{C} \subset \widehat{M} \times \widetilde{N}$ then we have (see [37, p.12])

$$
\begin{array}{ll}
R_{a b c d}=\rho_{1} G_{a b c d}, & \rho_{1}=\frac{\widehat{\kappa}}{2} \\
R_{\alpha b c \beta}=\rho_{2} G_{\alpha b c \beta}, & \rho_{2}=-\frac{\operatorname{tr}(T)}{4 F},
\end{array}
$$




$$
\begin{gathered}
R_{\alpha \beta \gamma \delta}=\rho_{3} G_{\alpha \beta \gamma \delta}, \quad \rho_{3}=\frac{1}{F}\left(\frac{\widetilde{\kappa}}{(n-3)(n-2)}-\frac{\Delta_{1} F}{4 F}\right), \\
S_{a b}=\mu_{1} g_{a b}, \quad \mu_{1}=\frac{1}{4 F}(2 F \widehat{\kappa}-(n-2) \operatorname{tr}(T)), \\
S_{\alpha \beta}=\mu_{2} g_{\alpha \beta}, \quad \mu_{2}=\frac{1}{F}\left(\frac{\widetilde{\kappa}}{n-2}-\frac{\operatorname{tr}(T)}{2}-(n-3) \frac{\Delta_{1} F}{4 F}\right) .
\end{gathered}
$$

In the considered case if the equality (4.21) does not hold then $U_{S} \cap U_{C}=\widehat{M} \times \widetilde{N}$. Computing now $\mu_{1}, \mu_{2}$ and $\rho_{1}, \rho_{2}, \rho_{3}$ from Remark 4.3, in view of (4.18) and (4.20), we have

$$
\mu_{1}=-\frac{n-1}{4} D, \quad \mu_{2}=\frac{1}{F}\left(\frac{\widetilde{\kappa}}{n-2}-\frac{n-1}{4} F D-(n-3)\left(B_{2}^{2}-C B^{2}\right)\right) .
$$

Thus

$$
\mu_{2}-\mu_{1}=\frac{1}{F}\left(\frac{\widetilde{\kappa}}{n-2}-(n-3)\left(B_{2}^{2}-C B^{2}\right)\right)
$$

$$
\begin{gathered}
\rho_{1}=\frac{\widehat{\kappa}}{2}=-\frac{D}{4}=\rho_{2}, \quad \rho_{3}=\frac{1}{F(n-3)}\left(\frac{\widetilde{\kappa}}{n-2}-(n-3)\left(B_{2}^{2}-C B^{2}\right)-(n-3) \frac{F D}{4}\right), \\
\rho_{3}=\frac{1}{n-3}\left(\mu_{2}-\mu_{1}\right)-\frac{D}{4} .
\end{gathered}
$$

According to [37, Theorem 4.1] $\widehat{M} \times{ }_{F} \widetilde{N}$ is a Roter manifold, i.e. (2.9) is satisfied, with $\phi=\nu\left(\rho_{1}-2 \rho_{2}+\rho_{3}\right), \mu=\nu\left(\left(\rho_{2}-\rho_{3}\right) \mu_{1}+\left(\rho_{2}-\rho_{1}\right) \mu_{2}\right), \eta=\nu\left(\rho_{1} \mu_{2}^{2}-2 \rho_{2} \mu_{1} \mu_{2}+\rho_{3} \mu_{1}^{2}\right)$, where $\nu=\left(\mu_{2}-\mu_{1}\right)^{-2}$. Thus applying these results we obtain

$$
\phi=\frac{1}{(n-3)\left(\mu_{2}-\mu_{1}\right)}, \quad \mu=-\frac{\mu_{1}}{(n-3)\left(\mu_{2}-\mu_{1}\right)}, \quad \eta=\rho_{1}+\frac{\mu_{1}^{2}}{(n-3)\left(\mu_{2}-\mu_{1}\right)} .
$$

Substituting these equalities to (2.13) we get

$$
L_{R}=-\frac{D}{4}=\frac{\widehat{\kappa}}{2}=\widehat{\kappa}_{G} .
$$

We see that $\widehat{M} \times_{F} \widetilde{N}$ is a Roter type manifold, in particular pseudosymmetric manifold of constant type, and admits geodesic mapping into $\widehat{\widehat{M}} \times \bar{F} \widetilde{N}$, so $\widehat{\widehat{M}} \times \bar{F} \widetilde{N}$ is pseudosymmetric manifold of constant type (see Theorem 3.1). We would like to show that it is also a Roter type manifold. First we compute the components of the tensor $\bar{T}$.

We observe, in view of (3.1), (3.5), (4.1) and (3.6) that

$$
\begin{aligned}
& \bar{\Gamma}_{11}^{1}=\Gamma_{11}^{1}+2 \psi_{1}=\frac{\mathfrak{a}^{\prime}}{2 \mathfrak{a}}-\frac{q \mathfrak{b}^{\prime}}{1+q \mathfrak{b}}, \\
& \bar{\Gamma}_{12}^{2}=\Gamma_{12}^{2}+\psi_{1}=\frac{\mathfrak{b}^{\prime}}{2 \mathfrak{b}(1+q \mathfrak{b})} \\
& \bar{\Gamma}_{22}^{1}=\Gamma_{22}^{1}=-\frac{\mathfrak{b}^{\prime}}{2 \mathfrak{a}} .
\end{aligned}
$$


Taking into account (4.12) and (4.17) we have

$$
\bar{F}\left(x^{1}, x^{2}\right)=\frac{p \mathfrak{b}\left(x^{1}\right) B^{2}\left(x^{2}\right)}{1+q \mathfrak{b}\left(x^{1}\right)},
$$

so $\bar{F}_{1}=\partial_{1} \bar{F}=\frac{\mathfrak{b}^{\prime}}{(1+q \mathfrak{b})^{2}} p B^{2}, \bar{F}_{2}=\partial_{2} \bar{F}=\frac{p \mathfrak{b}}{1+q \mathfrak{b}} 2 B B_{2}$ and

$$
\partial_{2} \bar{F}_{2}=\frac{p \mathfrak{b}}{1+q \mathfrak{b}} 2\left(B_{2}^{2}+B B_{22}\right)=\frac{p \mathfrak{b}}{1+q \mathfrak{b}} 2\left(B_{2}^{2}+C B^{2}\right),
$$

in virtue of (4.13). Next, using (4.30) and (4.15) we obtain

$$
\begin{gathered}
\bar{T}_{12}=\bar{\nabla}_{1} \bar{F}_{2}-\frac{1}{2 \bar{F}} \bar{F}_{1} \bar{F}_{2}=\partial_{1} \bar{F}_{2}-\bar{F}_{2} \bar{\Gamma}_{12}^{2}-\frac{1}{2 \bar{F}} \bar{F}_{1} \bar{F}_{2}=0, \\
\bar{T}_{22}=\bar{\nabla}_{2} \bar{F}_{2}-\frac{1}{2 \bar{F}}\left(\bar{F}_{2}\right)^{2}=\partial_{2} \bar{F}_{2}-\bar{F}_{1} \bar{\Gamma}_{22}^{1}-\frac{1}{2 \bar{F}}\left(\bar{F}_{2}\right)^{2}=\frac{D+4 q C}{2 p} \frac{p \mathfrak{b}}{1+q \mathfrak{b}} \bar{F}=\frac{D+4 q C}{2 p} \bar{F} \bar{g}_{22}, \\
\bar{T}_{11}=\bar{\nabla}_{1} \bar{F}_{1}-\frac{1}{2 \bar{F}}\left(\bar{F}_{12}\right)^{2}=\partial_{1} \bar{F}_{1}-\bar{F}_{1} \bar{\Gamma}_{11}^{1}-\frac{1}{2 \bar{F}_{1}}\left(\bar{F}_{1}\right)^{2}=\frac{p B^{2}}{(1+q \mathfrak{b})^{2}}\left(\mathfrak{b}^{\prime \prime}-\frac{1+2 q \mathfrak{b}}{2 \mathfrak{b}(1+q \mathfrak{b})}\left(\mathfrak{b}^{\prime}\right)^{2}-\frac{\mathfrak{a}^{\prime} \mathfrak{b}^{\prime}}{2 \mathfrak{a}}\right) .
\end{gathered}
$$

But, in virtue of (4.14) $\mathfrak{b}^{\prime \prime}-\frac{\mathfrak{a}^{\prime} \mathfrak{b}^{\prime}}{2 \mathfrak{a}}=\frac{\left(\mathfrak{b}^{\prime}\right)^{2}}{\mathfrak{b}}+2 \mathfrak{a} C$, and

$\bar{T}_{11}=\frac{p B^{2}}{(1+q \mathfrak{b})^{2}}\left(\frac{\left(\mathfrak{b}^{\prime}\right)^{2}}{2 \mathfrak{b}(1+q \mathfrak{b})}+2 \mathfrak{a} C\right)=\frac{p\left(\mathfrak{b}^{\prime}\right)^{2}}{\mathfrak{b}(D \mathfrak{b}-4 C)(1+q \mathfrak{b})^{2}} \frac{p \mathfrak{b} B^{2}}{1+q \mathfrak{b}} \frac{D+4 q C}{2 p}=\bar{g}_{11} \bar{F} \frac{D+4 q C}{2 p}$.

Thus we see that the following equality holds

$$
\bar{T}_{a b}=\frac{D+4 q C}{2 p} \bar{F} \bar{g}_{a b} .
$$

From the first equation of (3.3), using (4.1) and (3.5), we find $\psi_{11}=\partial_{1} \psi_{1}-\psi_{1} \Gamma_{11}^{1}-\left(\psi_{1}\right)^{2}$ and in virtue of (3.6), (4.14) and (4.15), we get

$$
\psi_{11}=\frac{q\left(\mathfrak{b}^{\prime}\right)^{2}\left(4 C-q D \mathfrak{b}^{2}-2 D \mathfrak{b}\right)}{4 \mathfrak{b}(1+q \mathfrak{b})^{2}(D \mathfrak{b}-4 C)} .
$$

Similarly, $\psi_{22}=\partial_{2} \psi_{2}-\psi_{1} \Gamma_{12}^{1}-\left(\psi_{2}\right)^{2}=-\psi_{1} \Gamma_{12}^{1}$ and

$$
\psi_{22}=-\frac{q \mathfrak{b}(D \mathfrak{b}-4 C)}{4(1+q \mathfrak{b})} .
$$

Using (3.5) we have $\Gamma_{\alpha \beta}^{1}=-\frac{1}{2} \widehat{g}^{11} F_{1} \widetilde{g}_{\alpha \beta}=-\frac{1}{2 \mathfrak{a}} F_{1} \widetilde{g}_{\alpha \beta}$.

Substituting this equality into $\psi_{\alpha \beta}=\partial_{\beta} \psi_{\alpha}-\psi_{1} \Gamma_{\alpha \beta}^{1}$ we obtain

$$
\psi_{\alpha \beta}=-\frac{q \mathfrak{b} B^{2}(D \mathfrak{b}-4 C)}{4(1+q \mathfrak{b})} \widetilde{g}_{\alpha \beta} .
$$

In the same manner we easily get

$$
\psi_{12}=0, \quad \psi_{a \alpha}=0 .
$$

Starting with (3.2) we calculate the components of $\bar{S}$.

Since $\bar{S}_{11}=S_{11}-(n-1) \psi_{11}$, so using (4.22), (4.32) and (4.15) we have

$$
\bar{S}_{11}=-\frac{n-1}{4 p}(D+4 q C) \bar{g}_{11} \text {. }
$$


Similarly, using (4.22) and (4.33) we obtain

$$
\bar{S}_{22}=-\frac{n-1}{4 p}(D+4 q C) \bar{g}_{22} .
$$

Now the last two equations and $\bar{S}_{12}=0$ yield

$$
\bar{S}_{a b}=-\frac{n-1}{4 p}(D+4 q C) \bar{g}_{a b} .
$$

Taking into account (4.23) and (4.34) we get

$$
\bar{S}_{\alpha \beta}=\left(\frac{\widetilde{\kappa}}{n-2}+(n-3)\left(C B^{2}-B_{2}^{2}\right)-\frac{n-1}{4} \mathfrak{b} B^{2} \frac{D+4 q C}{1+q \mathfrak{b}}\right) \frac{1}{\bar{F}} \bar{g}_{\alpha \beta} .
$$

Finally, in view of (4.35) we have

$$
\bar{S}_{a \alpha}=0
$$

On the other hand (4.3) leads to

$$
\bar{S}_{a b}=\overline{\widehat{S}}_{a b}-\frac{n-2}{2 \bar{F}} \bar{T}_{a b} .
$$

Thus substituting into this equality (4.36) and (4.31) we obtain

$$
\overline{\widehat{S}}_{a b}=-\frac{D+4 q C}{4 p} \bar{g}_{a b},
$$

which implies

$$
\bar{\kappa}=-\frac{D+4 q C}{2 p}
$$

Equalities (4.36), (4.37) and (4.38) imply that $\widehat{\widehat{M}} \times_{\bar{F}} \widetilde{N}$ cannot be quasi-Einsteinian and will be Einsteinian if and only if

$$
-\frac{n-1}{4 p}(D+4 q C) \frac{p \mathfrak{b} B^{2}}{1+q \mathfrak{b}}=\frac{\widetilde{\kappa}}{n-2}+(n-3)\left(C B^{2}-B_{2}^{2}\right)-\frac{n-1}{4 p} \mathfrak{b} B^{2} \frac{D+4 q C}{1+q \mathfrak{b}},
$$

which reduces to the equality (4.21).

According to Remark $4.2 \widehat{\widehat{M}} \times_{\bar{F}} \widetilde{N}$ is conformally flat if and only if $\bar{\rho}_{0}=0$, i.e.

$$
\frac{\widehat{\kappa}}{2} \bar{F}+\frac{\widetilde{\kappa}}{(n-3)(n-2)}+\frac{\operatorname{tr}(\bar{T})}{2}=\frac{\bar{\Delta}_{1} \bar{F}}{4 \bar{F}} .
$$

Substituting (4.39) into (4.31) we have $\bar{T}=-\bar{\kappa} \bar{F} \bar{g}$ which gives $\operatorname{tr}(\bar{T})=-2 \bar{\kappa} \bar{F}$ and

$$
\frac{\bar{\kappa}}{2} \bar{F}+\frac{\operatorname{tr}(\bar{T})}{2}=-\frac{\bar{\kappa}}{2} \bar{F}=\frac{\mathfrak{b} B^{2}(D+4 q C)}{4(1+q \mathfrak{b})} .
$$

Next, since $\bar{\Delta}_{1} \bar{F}=\frac{1}{\bar{g}_{11}}\left(\bar{F}_{1}\right)^{2}+\frac{1}{\bar{g}_{22}}\left(\bar{F}_{2}\right)^{2}$, so using (4.15) we easily derive that

$$
\frac{\bar{\Delta}_{1} \bar{F}}{4 \bar{F}}=B_{2}^{2}+\frac{D \mathfrak{b}-4 C}{1+q \mathfrak{b}} B^{2}
$$


Thus the equality (4.40) takes the form

$$
\frac{\widetilde{\kappa}}{(n-3)(n-2)}=-\frac{\mathfrak{b} B^{2}(D+4 q C)}{4(1+q \mathfrak{b})}+B_{2}^{2}+\frac{(D \mathfrak{b}-4 C) B^{2}}{1+q \mathfrak{b}}=B_{2}^{2}-C B^{2}
$$

i.e. the equality (4.21). Therefore, if the equality (4.21) does not hold then $U_{\bar{S}} \cap U_{\bar{C}}=\widehat{\widehat{M}} \times \widetilde{N}$. Applying Remark 4.3 to the warped product $\widehat{\widehat{M}} \times_{\bar{F}} \widetilde{N}$ and using earlier results we have

so,

$$
\begin{gathered}
\bar{\rho}_{1}=\frac{\overline{\widehat{\kappa}}}{2}=\bar{\rho}_{2}, \\
\bar{\mu}_{1}=\frac{n-1}{2} \bar{\kappa}, \quad \bar{\mu}_{2}=\frac{1}{\bar{F}} \frac{\widetilde{\kappa}}{n-2}+\frac{1}{\bar{F}}(n-3)\left(C B^{2}-B_{2}^{2}\right)+\frac{n-1}{2} \bar{\kappa}
\end{gathered}
$$

$$
\begin{gathered}
\bar{\mu}_{2}-\bar{\mu}_{1}=\frac{1}{\bar{F}}\left(\frac{\widetilde{\kappa}}{n-2}+(n-3)\left(C B^{2}-B_{2}^{2}\right)\right) \\
\bar{\rho}_{3}=\frac{1}{\bar{F}}\left(\frac{\widetilde{\kappa}}{(n-2)(n-3)}+\bar{F} \frac{\bar{\kappa}}{2}+C B^{2}-B_{2}^{2}\right)=\frac{1}{n-3}\left(\bar{\mu}_{2}-\bar{\mu}_{1}\right)+\frac{\bar{\kappa}}{2} .
\end{gathered}
$$

According to [37, Theorem 4.1] $\widehat{\widehat{M}} \times \bar{F} \widetilde{N}$ is a Roter manifold, i.e.

$$
\bar{R}=\frac{\bar{\phi}}{2} \bar{S} \wedge \bar{S}+\bar{\mu} \bar{g} \wedge \bar{S}+\frac{\bar{\eta}}{2} \bar{g} \wedge \bar{g}
$$

with $\bar{\phi}=\bar{\nu}\left(\bar{\rho}_{1}-2 \bar{\rho}_{2}+\bar{\rho}_{3}\right), \bar{\mu}=\bar{\nu}\left(\left(\bar{\rho}_{2}-\bar{\rho}_{3}\right) \bar{\mu}_{1}+\left(\bar{\rho}_{2}-\bar{\rho}_{1}\right) \bar{\mu}_{2}\right), \bar{\eta}=\bar{\nu}\left(\bar{\rho}_{1} \bar{\mu}_{2}^{2}-2 \bar{\rho}_{2} \bar{\mu}_{1} \bar{\mu}_{2}+\bar{\rho}_{3} \bar{\mu}_{1}^{2}\right)$, where $\bar{\nu}=\left(\bar{\mu}_{2}-\bar{\mu}_{1}\right)^{-2}$. Applying calculated expressions for $\bar{\rho}_{1}, \bar{\rho}_{2}, \bar{\rho}_{3}$ we obtain

$$
\bar{\phi}=\frac{1}{(n-3)\left(\bar{\mu}_{2}-\bar{\mu}_{1}\right)}, \quad \bar{\mu}=-\frac{\bar{\mu}_{1}}{(n-3)\left(\bar{\mu}_{2}-\bar{\mu}_{1}\right)}, \quad \bar{\eta}=\bar{\rho}_{1}+\frac{\bar{\mu}_{1}^{2}}{(n-3)\left(\bar{\mu}_{2}-\bar{\mu}_{1}\right)} .
$$

Substituting these equalities into (2.13) we get

$$
L_{\bar{R}}=-\frac{D+4 q C}{4 p}=\frac{\bar{\kappa}}{2}=\bar{\kappa}_{G} .
$$

Thus we have proved the following.

Theorem 4.1. Let $(\widehat{M}, \widehat{g})$ be a 2-dimensional manifold with a metric $\widehat{g}$ given by

$$
\widehat{g}_{11}=\mathfrak{a}\left(x^{1}\right), \quad \widehat{g}_{22}=\mathfrak{b}\left(x^{1}\right), \quad \widehat{g}_{12}=0,
$$

where

$$
\mathfrak{a}=\frac{\left(\mathfrak{b}^{\prime}\right)^{2}}{\mathfrak{b}(D \mathfrak{b}-4 C)}, C, D \in \mathbb{R}
$$

Next let $(\tilde{N}, \widetilde{g})$ be an $(n-2)$-dimensional, $n \geqslant 4$, semi-Riemannian space of constant curvature, when $n \geqslant 5$ and let $\widehat{M} \times_{F} \widetilde{N}$ be the warped product manifold with warping function

$$
F=F\left(x^{1}, x^{2}\right)=\mathfrak{b}\left(x^{1}\right) B^{2}\left(x^{2}\right),
$$

where $B$ is a function described in Remark 4.1 such that the equality 4.21) does not hold. Then $\widehat{M} \times_{F} \widetilde{N}$ is a Roter type manifold which admits a non-trivial geodesic mapping onto a warped 
product manifold $\widehat{\widehat{M}} \times_{\bar{F}} \widetilde{N}$, where $(\overline{\widehat{M}}, \overline{\widehat{g}})$ is a manifold geodesically related to $(\widehat{M}, \widehat{g})$ with a metric $\bar{g}$ given by

$$
\overline{\widehat{g}}_{11}=\frac{p \mathfrak{a}}{(1+q \mathfrak{b})^{2}}, \quad \overline{\widehat{g}}_{22}=\frac{p \mathfrak{b}}{1+q \mathfrak{b}}, \quad \overline{\widehat{g}}_{12}=0, p, q \in \mathbb{R}
$$

and warping function $\bar{F}=\frac{p}{1+q \mathfrak{b}} F$. Moreover, $\widehat{\widehat{M}} \times_{\bar{F}} \widetilde{N}$ is also a Roter type manifold.

Proposition 4.2. Under above assumptions we have

$$
L_{R}=-\frac{D}{4}, \quad L_{\bar{R}}=-\frac{D+4 q C}{4 p},
$$

so both manifolds $(M, g)$ and $(\bar{M}, \bar{g})$ are pseudosymmetric of constant type. Moreover we have

$$
L_{R}-\frac{\kappa}{n(n-1)}=\frac{p}{1+q \mathfrak{b}}\left(L_{\bar{R}}-\frac{\bar{\kappa}}{n(n-1)}\right) .
$$

Proof. The equalities (4.29) and (4.41) give the first part of the assertion. Using (4.24), (4.25) and (4.26) we obtain

$$
\begin{aligned}
\kappa & =g^{a b} S_{a b}+g^{\alpha \beta} S_{\alpha \beta}=g^{a b} g_{a b} \mu_{1}+g^{\alpha \beta} g_{\alpha \beta} \mu_{2}=2 \mu_{1}+(n-2) \mu_{2}= \\
& =-\frac{2(n-1) D}{4}+\frac{n-2}{F}\left(\frac{\widetilde{\kappa}}{n-2}-\frac{n-1}{4} D F-(n-3)\left(B_{2}^{2}-C B^{2}\right)\right)= \\
& =-\frac{n(n-1)}{4} D+\frac{\widetilde{\kappa}}{F}-\frac{(n-3)(n-2)}{F}\left(B_{2}^{2}-C B^{2}\right)
\end{aligned}
$$

and in virtue of (4.29) we have

$$
\kappa=n(n-1) L_{R}+\frac{\widetilde{\kappa}}{F}-\frac{(n-3)(n-2)}{F}\left(B_{2}^{2}-C B^{2}\right) .
$$

Similarly, using analogous equations for $(\bar{M}, \bar{g})$ we get

$$
\bar{\kappa}=n(n-1) L_{\bar{R}}+\frac{\widetilde{\kappa}}{\bar{F}}-\frac{(n-3)(n-2)}{\bar{F}}\left(B_{2}^{2}-C B^{2}\right) .
$$

Comparing two last relations we have

$$
L_{R}-\frac{\kappa}{n(n-1)}=\frac{\bar{F}}{F}\left(L_{\bar{R}}-\frac{\bar{\kappa}}{n(n-1)}\right),
$$

and taking into account (4.12) we obtaion (4.42).

Roter type manifolds satisfy various curvature conditions of pseudosymmetry type. We find formulas for the functions $L_{C}$ and $L$ in (2.15) and (2.14).

Taking into account (2.12) and (2.15) we get

$$
L_{C}=L_{R}-\frac{\kappa}{n-1}+\frac{1-(n-2) \mu}{(n-2) \phi} .
$$


Using now (4.28), (4.27) and (4.26), after standard calculation we obtain

$$
\frac{(n-2)^{2}}{n} L_{C}=L_{R}-\frac{\kappa}{n(n-1)} \text {. }
$$

Similarly (for $\bar{g}$ ) we have

$$
\frac{(n-2)^{2}}{n} L_{\bar{C}}=L_{\bar{R}}-\frac{\bar{\kappa}}{n(n-1)} .
$$

Thus, in virtue of (4.42) we get

$$
L_{C}=\frac{\bar{F}}{F} L_{\bar{C}}=\frac{p}{1+q \mathfrak{b}} L_{\bar{C}} .
$$

Taking into account (2.14), (4.28) and (4.26) we easily obtain $L=-(n-2) L_{R}$ and, similarly $\bar{L}=-(n-2) L_{\bar{R}}$. Thus we have the following.

Corollary 4.2. For manifolds $(M, g)$ and $(\bar{M}, \bar{g})$ satisfying assumptions of the Theorem 4.1 we have

(i) $C \cdot C=L_{C} Q(g, C), \bar{C} \cdot \bar{C}=L_{\bar{C}} Q(\bar{g}, \bar{C})$, where $L_{C}$ is given by 4 .43) and $L_{C}=(p /(1+q \mathfrak{b})) L_{\bar{C}}$.

(ii) $R \cdot R=Q(S, R)-(n-2) L_{R} Q(g, C), \quad \bar{R} \cdot \bar{R}=Q(\bar{S}, \bar{R})-(n-2) L_{\bar{R}} Q(\bar{g}, \bar{C})$.

As we mentioned at the end of Section 1, we continue investigations of geodesic mappings in Roter spaces and, for example, we obtained

Remark 4.4. [34] Let $(M, g)$ be a pseudosymmetric non-semi-symmetric semi-Riemannian manifold admitting a non-trivial geodesic mapping onto a Roter space $(\bar{M}, \bar{g})$. Then we have the following

$$
(\bar{\kappa} \bar{\phi}+n \bar{\mu}) B-(\operatorname{tr}(B) \bar{\phi}+\operatorname{tr}(\psi) \bar{\mu}) \bar{S}+\left(\bar{\kappa} \bar{\mu}-n\left(L_{\bar{R}}-\bar{\eta}\right)\right) \psi+\left(\operatorname{tr}(\psi)\left(L_{\bar{R}}-\bar{\eta}\right)-\operatorname{tr}(B) \bar{\mu}\right) \bar{g}=0
$$

where $\psi$ and $B$ are (0,2)-tensors with components given by (3.3) and $B_{m k}=\psi_{m r} \bar{S}_{k}^{r}$, respectively.

Moreover, we found the sufficient conditions for the manifold $(M, g)$ to be also a Roter space.

Acknowledgements. The first named author is supported by a grant of the Wrocław University of Environmental and Life Sciences (Poland).

\section{REFERENCES}

[1] K. Arslan, R. Deszcz, R. Ezentaş, M. Hotloś and C. Murathan, On generalized Robertson-Walker spacetimes satisfying some curvature condition, Turkish J. Math. 38 (2014), 353-373.

[2] A.L. Besse, Einstein Manifolds, Ergeb. Math. Grenzgeb., 3. Folge, Bd. 10. Springer-Verlag, Berlin, Heidelberg, New York, 1987.

[3] E. Boeckx, O. Kowalski, L. Vanhecke, Riemannian manifolds of connulity two, World Sci.Publ., River edge, NJ, 1996.

[4] T.E. Cecil, P.J. Ryan, Geometry of Hypersurfaces, Springer Monographs in Mathematics, Springer New York Heidelberg Dodrecht London, 2015.

[5] B.-Y. Chen, Pseudo-Riemannian Geometry, $\delta$-Invariants and Applications, World Scientific, 2011.

[6] B.-Y. Chen, Differential Geometry of Warped Product Manifolds and Submanifolds, World Scientific, 2017. 
[7] B.-Y. Chen, Classification of torqued vector fields and its applications to Ricci solitons, Kragujevac J. Math. 41 (2017), 239-250.

[8] J. Chojnacka-Dulas, R. Deszcz, M. Głogowska and M. Prvanović, On warped products manifolds satisfying some curvature conditions, J. Geom. Phys. 74 (2013), 328-341.

[9] S. Decu, M. Petrović-Torgašev, A. Šebeković and L. Verstraelen, On the Roter type of Wintgen ideal submanifolds, Rev. Roumaine Math. Pures Appl. 57 (2012), 75-90.

[10] F. Defever, R. Deszcz, A note on geodesic mappings of pseudosymmetric Riemannian manifolds, Colloq. Math. 62 (1991), 313-319.

[11] F. Defever, R. Deszcz, Some results on geodesic mappings of Riemannian manifolds satisfying the condition $R \cdot R=Q(S, R)$, Periodica Math. Hungarica 29(3) (1994), 267-276.

[12] F. Defever, R. Deszcz and M. Prvanović, On warped product manifolds satisfying some curvature condition of pseudosymmetry type, Bull. Greek Math. Soc. 36 (1994), 43-62.

[13] J. Deprez, R. Deszcz and L. Verstraelen, Examples of pseudosymmetric conformally flat warped products, Chinese J. Math. 17 (1989), 51-65.

[14] A. Derdziński and W. Roter, Some properties of conformally symmetric manifolds which are not Riccirecurrent, Tensor (N.S.) 34 (1980), 11-20.

[15] R. Deszcz, On pseudosymmetric spaces, Bull. Soc. Math. Belg.44 (1992), Sér. A, Fasc. 1, 1-34.

[16] R. Deszcz, On some Akivis-Goldberg type metrics, Publ. Inst. Math. (Beograd) (N.S.) 74 (88) (2003), $71-83$.

[17] R. Deszcz, M. Głogowska, H. Hashiguchi, M. Hotloś and M. Yawata, On semi-Riemannian manifolds satisfying some conformally invariant curvature condition, Colloq. Math. 131 (2013), 149-170.

[18] R. Deszcz, M. Głogowska, M. Hotloś, and K. Sawicz, A Survey on Generalized Einstein Metric Conditions, Advances in Lorentzian Geometry: Proceedings of the Lorentzian Geometry Conference in Berlin, AMS/IP Studies in Advanced Mathematics 49, S.-T. Yau (series ed.), M. Plaue, A.D. Rendall and M. Scherfner (eds.), $2011,27-46$.

[19] R. Deszcz, M. Głogowska, M. Hotloś and Z. SSentürk, On certain quasi-Einstein semisymmetric hypersurfaces, Ann. Univ. Sci. Budapest. Eötvös Sect. Math. 41 (1998), 151-164.

[20] R. Deszcz, M. Głogowska, M. Hotloś, J. Jełowicki and G. Zafindratafa, Curvature properties of some warped product manifolds, poster, conference Differential Geometry, Banach Conference Center at Bedlewo, Poland, June 19 - June 24, 2017.

[21] R. Deszcz, M. Głogowska, M. Hotloś and G. Zafindratafa, On some curvature conditions of pseudosymmetry type, Period. Math. Hung. 70 (2015), 153-170.

[22] R. Deszcz, M. Głogowska, M. Hotloś and G. Zafindratafa, Hypersurfaces in spaces forms satisfying some curvature conditions, J. Geom. Phys. 99 (2016), 218-231.

[23] R. Deszcz, M. Głogowska, J. Jełowicki and G. Zafindratafa, Curvature properties of some class of warped product manifolds, Int. J. Geom. Meth. Modern Phys. 13 (2016), 1550135 (36 pages).

[24] R. Deszcz, M. Głogowska, M. Petrović-Torgašev and L. Verstraelen, On the Roter type of Chen ideal submanifolds, Results. Math. 59 (2011), 401-413.

[25] R. Deszcz, M. Głogowska, M. Petrović-Torgašev and L. Verstraelen, Curvature properties of some class of minimal hypersurfaces in Euclidean spaces, Filomat 29 (2015), 479-492.

[26] R. Deszcz, M. Głogowska, M. Plaue, K. Sawicz and M. Scherfner, On hypersurfaces in space forms satisfying particular curvature conditions of Tachibana type, Kragujevac J. Math. 35 (2011), 223-247.

[27] R. Deszcz, S. Haesen and L. Verstraelen, On natural symmetries, Topics in Differential Geometry, Ch. 6, Editors A. Mihai, I. Mihai and R. Miron, Editura Academiei Române, 2008, 249-308.

[28] R.Deszcz, M. Hotloś, On geodesic mappings in pseudo-symmetric manifolds, Bull. Inst. Math. Acad. Sinica 16(3) (1988), 251-262.

[29] R.Deszcz, M. Hotloś, Remarks on Riemannian manifolds satisfying certain curvature condition imposed on the Ricci tensor, Prace Nauk. Pol. Szczec. 11 (1988), 23-34.

[30] R.Deszcz, M. Hotloś, Notes on pseudo-symmetric manifolds admitting special geodesic mappings, Soochow J. Math. 15 (1989), 19-27. 
[31] R. Deszcz and M. Hotloś, On certain subclass of pseudosymmetric manifolds, Publ. Math. Debrecen 53 (1998), 29-48.

[32] R. Deszcz and M. Hotloś, On hypersurfaces with type number two in space forms, Ann. Univ. Sci. Budapest. Eötvös Sect. Math. 46 (2003), 19-34.

[33] R. Deszcz and M. Hotloś, On some pseudosymmetry type curvature condition, Tsukuba J. Math. 27 (2003) $13-30$.

[34] R. Deszcz and M. Hotloś, On geodesically related Roter spaces, to appear.

[35] R. Deszcz, M. Hotloś, J. Jełowicki, H. Kundu, and A.A. Shaikh, Curvature properties of Gödel metric. Int. J. Geom. Meth. Modern Phys. 11 (2014), 1450025 (20 pages).

[36] R. Deszcz, M. Hotloś and Z. Şentürk, On curvature properties of certain quasi-Einstein hypersurfaces, Int. J. Math. 23 (2012), 1250073 (17 pages).

[37] R. Deszcz and D. Kowalczyk, On some class of pseudosymmetric warped products, Colloq. Math. 97 (2003), $7-22$.

[38] R. Deszcz, M. Petrović-Torgašev, L. Verstraelen and G. Zafindratafa, On Chen ideal submanifolds satisfying some conditions of pseudo-symmetry type, Bull. Malaysian Math. Sci. Soc. 39 (2016), 103-131.

[39] R. Deszcz, M. Plaue and M. Scherfner, On Roter type warped products with 1-dimensional fibres, J. Geom. Phys. 69 (2013), 1-11.

[40] R. Deszcz and M. Scherfner, On a particular class of warped products with fibres locally isometric to generalized Cartan hypersurfaces, Colloq. Math. 109 (2007), 13-29.

[41] R. Deszcz, L. Verstraelen and L. Vrancken, The symmetry of warped product spacetimes, Gen. Relativ. Gravit. 23 (1991), 671-681.

[42] R. Deszcz and Ş. Yaprak, Curvature properties of certain pseudosymmetric manifolds, Publ. Math. Debrecen 45 (1994), 333-345.

[43] M. Głogowska, Curvature conditions on hypersurfaces with two distinct principal curvatures, in: Banach Center Publ. 69, Inst. Math., Polish Acad. Sci. 133-143, 2005.

[44] M. Głogowska, On Roter type manifolds, in: Pure and Applied Differential Geometry - PADGE 2007, Shaker Verlag, Aachen, 2007, 114-122.

[45] M. Głogowska, On quasi-Einstein Cartan type hypersurfaces, J. Geom. Phys. 58 (2008), 599-614.

[46] J.B. Griffiths and J. Podolský, Exact Space-Times in Einsteins General Relativity, Cambridge Univ. Press, 2009.

[47] S. Haesen and L. Verstraelen, Properties of a scalar curvature invariant depending on two planes, Manuscripta Math. 122 (2007), 59-72.

[48] S. Haesen and L. Verstraelen, Natural intrinsic geometrical symmetries, SIGMA 5 (2009), 086 (14 pages).

[49] I. Hinterleitner, Selected special vector fields and mappings in Riemannian geometry, J. Appl. Math. $1: 2$ (2008), 30-37.

[50] I. Hinterleitner, Special mappings of equidistant spaces, J. Appl. Math. 2 (2008), 31-36.

[51] D. Kowalczyk, On some class of semisymmetric manifolds, Soochow J. Math. 27 (2001), 445-461.

[52] D. Kowalczyk, On the Reissner-Nordström-de Sitter type spacetimes, Tsukuba J. Math. 30 (2006), 363-381.

[53] O. Kowalski and M. Sekizawa, Pseudo-symmetric spaces of constant type in dimension three - elliptic spaces, Rend. Mat. Appl., VII Ser., 17 (1997), 477-512.

[54] O. Kowalski and M. Sekizawa, Pseudo-symmetric spaces of constant type in dimension three - non-elliptic spaces, Bull. Tokyo Gakugei Univ. (4) 50 (1998), 1-28.

[55] Ü. Lumiste, Semiparallel Submanifolds in Space Forms, Springer Science + Business Media, New York, LLC 2009.

[56] J. Mikeš, Geodesic mappings of special Riemannian spaces, in: Topics in Differential Geometry (Hajduszoboszló 1984), Colloq. Math. Soc. János Bolyai 46, Vol. II, North-Holland, Amsterdam 1988, 793-813.

[57] J. Mikeš, E. Stepanova, A. Vanžurová and et. al., Differential geometry of special mappings, Palacký Univ. Press, Olomouc, 2015.

[58] C. Murathan, K. Arslan, R. Deszcz, R. Ezentaş and C. Özgür, On a certain class of hypersurfaces of semi-Euclidean spaces, Publ. Math. Debrecen 58 (2001), 587-604. 
[59] B. O'Neill, Semi-Riemannian Geometry with Applications to Relativity, Academic Press, New York - London, 1983.

[60] W. Roter, A note on second order recurrent spaces, Bull. Acad. Polon. Sci., Sér. sci. math., astr. et phys., 10 (1964), 621-626.

[61] A.A. Shaikh, R. Deszcz, M. Hotloś, J. Jełowicki, and H. Kundu, On pseudosymmetric manifolds, Publ. Math. Debrecen 86 (2015), 433-456.

[62] N.S. Sinjukov, Geodesic mappings of Riemannian spaces (in Russian), Moscow, 1979.

[63] Z. Stuchlik and S. Hledik, Properties of the Reissner-Nordström spacetimes with a nonzero cosmological constant, Acta Phys. Slovaca 52 (2002), 363-407.

[64] Z.I. Szabó, Structure theorems on Riemannian spaces satisfying $R(X, Y) \cdot R=0$. I. The local version, J. Differential Geom. 17 (1982), 531-582.

[65] P. Venzi, On geodesic mappings in Riemannian or pseudo-Riemannian manifolds, Tensor (N.S.) 32 (1978), 193-198.

[66] L. Verstraelen, Comments on the pseudo-symmetry in the sense of Ryszard Deszcz, in: Dillen e.a.(eds.) Geometry and Topology of Submanifolds, VI, World Sci., Singapore, 1994, 119-209.

[67] L. Verstraelen, Natural extrinsic geometrical symmetries - an introduction -, in: Recent Advances in the Geometry of Submanifolds Dedicated to the Memory of Franki Dillen (1963-2013), Contemporary Mathematics, 674 (2016), 5-16.

[68] L. Verstraelen, Foreword, in: B.-Y. Chen, Differential Geometry of Warped Product Manifolds and Submanifolds, World Scientific, 2017, vii-xxi.

Ryszard Deszcz

Department of Mathematics

Wrocław Univeristy of Environmental and Life Sciences

Grunwaldzka 53, 50-357 Wrocław, Poland

E-mail: Ryszard.Deszcz@upwr.edu.pl

Marian Hotloś

Department of Applied Mathematics

Wrocław Univeristy of Science and Technology

Wybrzeże Wyspiańskiego 27, 50-370 Wrocław, Poland

E-mail: Marian.Hotlos@pwr.edu.pl 